\title{
O tamanho do ovo não prediz o desenvolvimento físico de avestruzes (Struthio camelus) aos quinze dias de idade
}

\author{
The size of the egg does not predict the physical development of ostriches (Struthio camelus) \\ at fifteen days old
}

Adriano Braga Brasileiro de Alvarenga' ${ }^{\mathrm{I}}$ Vanner Boere ${ }^{\mathrm{II}}$

\section{RESUMO}

O tamanho dos ovos está relacionado com a sobrevivência e o crescimento de filhotes de aves precociais. A inter-relação entre ovo e filhote pode ser importante para a produção animal, fornecendo subsídios para decisões estratégicas de economia, manejo e bem-estar animal. Este estudo analisou a relação entre o peso dos ovos antes da incubação e o desenvolvimento de filhotes de avestruzes $(N=48)$ aos quinze dias de idade. Os filhotes foram criados em grupos, em berçários, com alimentação comercial e sem cuidado parental. Nos filhotes, mensurou-se o peso, a altura da cernelha e o comprimento do tarso-metatarso. Houve uma significativa diferença nos pesos dos ovos, mas as medidas corporais equivalentes dos filhotes não foram estatisticamente diferentes. Em concordância com estudos em outras espécies de aves, o peso do ovo não parece influenciar o crescimento dos filhotes de avestruzes até os 15 dias de vida, em avestruzes.

Palavras-chave: Avestruz, Struthio camelus, peso do ovo, peso dos filhotes, crescimento.

\section{ABSTRACT}

The size of the eggs is related to survival and chick growth in precocial birds. The interrelationship between egg and chick development can be important for the animal production, supplying data for strategic decisions of economy, handling and animal welfare. This study analyzed the relation between the weight of the eggs and the development of ostrich chicks $(N=48)$ at age of fifteen days old. The chicks were kept in indoor nurseries, in groups, without parental care, and fed commercial feed. It was recorded the weight, the back height and the length of the tarsus-metatarsus. There was a significant difference in the weight of the eggs, but the correspondent body chicken measures were not statistically different. In agreement to the studies in other species of birds, the weight of the egg does not seem to influence the growth of the ostriches at fifteen days old.
Key words: Ostrich, Struthio camelus, egg weight, chick weight, growth.

\section{INTRODUÇÃO}

A morfologia e o peso do ovo são considerados importantes indícios da qualidade do embrião e da sobrevida dos filhotes de aves logo após o nascimento (NOLAN JR \& THOMPSON, 1978; BOLTON, 1991; HILL, 1995). As investigações com aves sugerem que ovos maiores podem possuir maior resistência térmica (RHYMER 1988), mais proteínas e lipídeos (NAGER et al., 2000; SLATTERY \& ALISAUSKAS, 1995) e originar filhotes com um maior desempenho motor (ANDERSON \& ALISAUSKAS, 2001).

As dimensões do ovo (diâmetro maior e menor) são fortemente correlacionadas com o peso (BLOMQVIST et al., 1997), sendo este último recomendado como medida de tamanho do ovo para aves ratitas (Struthioniformes), por ser um método rápido e confiável (DI CAMPOS et al., 2005). A maior parte do peso do ovo, logo após a postura, deve-se à quantidade de albúmen e à proporção de água (FINKLER et al., 1998; DZIALOWSKI \& SOTHERLAND, 2004). Essas características resultariam em uma relação positiva e significativa entre a dimensão do ovo, o seu tamanho (NOLAN JR \& THOMPSON, 1978; FINKLER et al., 1998; NAGER et al., 2000; DZIALOWSKI \& SOTHERLAND, 2004) e a

\footnotetext{
IDepartamento de Ciências Biológicas, Faculdade Alvorada, Brasília, DF, Brasil.

IIDepartamento de Ciências Fisiológicas (CFS), Instituto de Biologia, Universidade de Brasília (UnB), 70910-900, Brasília, DF, Brasil. E-mail: vanner@unb.br. Autor para correspondência.
} 
sobrevivência do filhote após a eclosão (WILLIAMS, 1994; LINDSTRÖM, 1999).

Em avestruzes, foi demonstrado que há uma alta correlação entre o peso do ovo e o peso do filhote ao nascer $\left(\mathrm{R}^{2}=0,84\right)$ e que esta relação vai decrescendo com o avanço dos dias (ZOCCARATO et al., 2004). GONZALES et al. (1999) observaram que o peso do ovo se correlacionava fortemente com o peso do filhote em avestruzes; porém, concluíram que ovos com pesos intermediários, e não os ovos com pesos maiores ou menores, obtiveram melhor índice de eclosão.

A qualidade do ovo, representada pelo seu tamanho e por seus constituintes, é, em parte, o componente de estratégias parentais altriciais e precociais. Em aves altriciais, o ovo representa um relativo menor investimento parental (da fêmea reprodutiva) quando comparado com o investimento no cuidado após a eclosão (REED et al., 1999). Em aves precociais, os filhotes estão em um estágio de desenvolvimento avançado ao eclodirem, embora possam depender do cuidado parental por um curto período. A independência do filhote precocial é, parcialmente, conseqüência de um aporte completo de nutrientes durante a fase embrionária, cujas reservas são proporcionais ao tamanho do ovo (BLOMQVIST et al., 1997; REED et al., 1999). Por conseguinte, existem fortes evidências de que o tamanho dos filhotes recémeclodidos esteja relacionado à capacidade de sobrevivência dos mesmos (WILLIAMS, 1994; LINDSTRÖM, 1999).

A avestruz é uma ave reconhecida como precocial (DEEMING \& AR, 1999) e apresenta cuidado parental por um curto período, realizado principalmente pelo macho. Além disso, o ovo é relativamente pequeno em relação à massa corporal adulta (RAHN et al., 1975), ao contrário de outras aves do grupo das ratitas.

As primeiras semanas de vida de avestruzes é uma fase que pode ser decisiva para a seleção de animais mais bem adaptados para produção ou reprodução em uma criação (ZOCCARATO et al., 2004; DI CAMPOS et al., 2005). Na estrutiocultura, há o pressuposto de que preservando as qualidades de integridade e viabilidade, os ovos maiores gerarão filhotes maiores.

A relação entre o peso dos ovos e as características físicas dos filhotes de avestruzes pode ser útil para a definição de estratégias de manejo na criação que resultem em melhores ganhos e padrões aceitáveis de bem-estar animal. Entretanto, um dos problemas na obtenção de dados básicos em estrutiocultura está na diversificação das condições em que são criados os avestruzes, entre locais com climas tão variados (ZOCCARATO et al., 2004). O objetivo deste estudo foi analisar a relação entre o peso inicial dos ovos e a ontogenia física de avestruzes até a primeira quinzena de idade, na região do Planalto Central, Distrito Federal.

\section{MATERIAL E MÉTODOS}

A coleta dos dados foi realizada entre outubro e novembro de 2004, na Fazenda Brasília Avestruz, situada no quilômetro 6 da rodovia DF - 290, localidade de Engenho das Lajes, Brasília-DF. A propriedade realiza a incubação dos ovos, a cria de filhotes e a comercialização de filhotes e adultos.

Foram utilizados 48 ovos, submetidos a pesagem e desinfecção. A incubação foi realizada artificialmente a uma temperatura média de $36,1^{\circ} \mathrm{C}$ e umidade relativa média de $48 \%$, em incubadora elétrica. Foram realizadas três ovoscopias, com a finalidade de identificarem-se os ovos férteis em desenvolvimento, os ovos não-férteis e os ovos com morte embrionária.

Após o nascimento, os filhotes foram marcados e mantidos durante duas semanas em um berçário suspenso e, posteriormente, transferidos para quatro baias de cria com piso de concreto. A identificação do sexo dos filhotes não foi realizada devido às dificuldades técnicas. $\mathrm{O}$ acesso à água foi irrestrito e a ração balanceada (valor energético: 10,36MJ $\mathrm{kg}^{-1}$ ) foi ofertada duas vezes ao dia, em comedouros coletivos.

As comparações com o peso dos ovos e sua influência no desenvolvimento são mais evidentes quanto mais cedo forem realizadas as medidas. Espera-se um maior impacto das condições do ovo e menor das condições ambientais nos primeiros dias após a eclosão (KRIST et al., 2004). Por isso, cada filhote foi pesado e submetido a mensurações morfométricas (comprimento do tarso-metatarso direito, tarso-metatarso esquerdo e altura da cernelha) aos 15 dias de idade. A pesagem dos animais ocorreu em jejum, com o uso de uma balança eletrônica (Toledo, Brasil). Os comprimentos do tarso-metatarso direito e do esquerdo foram mensurados com uma fita métrica. A altura da cernelha foi medida com o auxílio de uma trena adaptada. Todos os procedimentos foram realizados no período matutino, das $6 \mathrm{~h}$ às $9 \mathrm{~h}$.

Para a análise das diferenças médias, seguiuse a metodologia de GONZALES et al. (1999), em que os ovos foram classificados em três categorias de pesos (ovo com peso menor, ovo com peso intermediário e ovo com peso maior). Os pesos dos ovos distribuídos nestas categorias e as medidas correspondentes dos filhotes foram analisados por uma ANOVA. Se necessário, aplicou-se um teste Tukey. Para se verificar 
a força da relação entre o peso inicial dos ovos e as medidas dos filhotes, construiu-se uma matriz de correlação de Spearman. As análises foram bicaudais com o nível de significância = 0,05.

\section{RESULTADOS}

O peso inicial do ovo obteve uma média $( \pm$ desviopadrão) de 1,34 $\pm 0,11 \mathrm{~kg}^{-1}$. Este parâmetro variou consideravelmente, oscilando entre o peso mínimo de $1,10 \mathrm{~kg}^{-1}$ e o peso máximo de $1,51 \mathrm{~kg}^{-1}$ ( $\mathrm{N}=48$ ovos). As médias do peso inicial dos ovos, do peso vivo e das mensurações morfométricas dos filhotes estão descritas na tabela 1 .

Devido à grande variância no peso inicial dos ovos, com mais do que um desvio padrão de diferença, realizou-se uma análise com os pesos iniciais dos ovos distribuídos em três categorias: peso menor, peso intermediário e peso maior (Tabela 2). Houve uma diferença significativa entre os pesos das três categorias de ovos (Tabela 2). Entretanto, as medidas morfométricas e o peso de cada filhote aos 15 dias de vida, correspondente ao ovo de origem, não foram estatisticamente diferentes.

Para se verificar a possível relação entre o peso inicial dos ovos distribuídos em categorias e as medidas morfométricas, construiu-se uma matriz de correlação de Spearman. Não houve correlação entre o peso inicial dos ovos e as medidas morfométricas dos filhotes (peso vivo; $r h o=0,11$; tarso metatarso direito, $r h o=0,03$; tarso-metatarso esquerdo, $r h o=0,04$; cernelha, $r h o=-0,03)$. Por outro lado, como esperado, o peso vivo, o comprimento do tarso-metatarso direito, o comprimento do tarso-metatarso esquerdo e a altura da cernelha de cada filhote foram fortemente correlacionados. Os valores de rho, das correlações

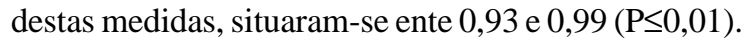

\section{DISCUSSÃO}

O peso dos ovos foi semelhante ao relatado em outros estudos de criação intensiva. Por exemplo,

Tabela 1 - Média do peso inicial dos ovos, do peso vivo e das mensurações morfométricas dos filhotes de avestruzes na primeira quinzena de vida, observados na Fazenda Brasília Avestruz, Brasília, DF.

\begin{tabular}{lc}
\hline Características morfométricas & Média \\
\hline Peso do ovo $(\mathrm{kg})$ & $1,34 \pm 0,11$ \\
Peso vivo $(\mathrm{kg})$ & $0,79 \pm 0,16$ \\
Tarso-metatarso direito $(\mathrm{cm})$ & $7,03 \pm 0,86$ \\
Tarso-metatarso esquerdo $(\mathrm{cm})$ & $6,99 \pm 0,86$ \\
Cernelha $(\mathrm{cm})$ & $21,86 \pm 3,00$ \\
\hline
\end{tabular}

Tabela 2 - Distribuição do peso dos ovos $\left(\mathrm{kg}^{-1}\right)$ em categorias: número de ovos $(\mathrm{N})$, média dos pesos \pm desvio padrão (DP) e valores extremos (mínimo e máximo), referentes aos filhotes de avestruzes na primeira quinzena de vida, observados na Fazenda Brasília Avestruz, Brasília, DF.

\begin{tabular}{lclcc}
\hline Categoria & N & Média & Mínimo & Máximo \\
\hline Peso menor & 12 & $1,18 \pm 0,04^{\mathrm{a}^{*}}$ & 1,10 & 1,20 \\
Peso intermediário & 26 & $1,35 \pm 0,05^{\mathrm{b}}$ & 1,26 & 1,40 \\
Peso maior & 10 & $1,47 \pm 0,04^{\mathrm{c}}$ & 1,41 & 1,51 \\
\hline
\end{tabular}

*Letra diferente dentro da mesma coluna indica diferença estatística $(\mathrm{P}<0,05)$.

na Itália, SUPERCHI et al. (2002) encontraram um peso médio dos ovos de avestruzes de $1,44 \mathrm{~kg}^{-1}$ e ZOCCARATO et al. (2004) obtiveram pesos médios que variaram de 1,31 a 1,41 $\mathrm{kg}^{-1}$. No México, um estudo encontrou pesos médios de ovos de avestruzes de 1,52 e 1,53 kg-1 , em duas estações reprodutivas (ESTRADA et al., 2006).

Houve uma grande variação no tamanho dos ovos estudados. Esta variação é comum em aves, pois dentro de um mesmo ninho, da mesma fêmea, pode haver variações extremas (REED et al., 1999; GÖTH \& EVANS, 2004). A razão peso maior/peso menor foi de 1,36, contrastando com os resultados de uma revisão realizada por CHRISTIANS (2002), na qual 49\% de 39 estudos revelaram valores entre 1,40 e 1,59. Esta razão provavelmente deve-se ao fator seleção, em que os criadores tendem a padronizar as características dos animais e de seus produtos.

Diversas variáveis podem interferir no tamanho dos ovos, como fase de postura, idade da fêmea e seqüência de postura (DEEMING \& AR, 1999; MAGRATH et al., 2003). Entretanto, há uma relação direta entre o tamanho do ovo e seus constituintes. Apesar da variação encontrada, a amostra foi de aves que sobreviveram até a primeira quinzena de vida. Essas aves não apresentaram diferenças morfométricas, sugerindo que o tamanho dos ovos não parece ter influenciado de forma determinante no desenvolvimento dos filhotes.

Ovos maiores possuem maior proteção contra microrganismos, devido à viscosidade e às enzimas proteolíticas presentes em um albúmen mais volumoso (SUPERCHI et al., 2002). Alguns autores explicitam que, mesmo na mesma ninhada, onde há diferenças substanciais no tamanho dos ovos, os ovos menores poderiam gerar filhotes menores sem que isso, contudo, seja necessariamente uma desvantagem. Isto porque filhotes menores seriam energeticamente menos exigentes nos primeiros dias de vida, o que seria uma vantagem para a sobrevivência delas em relação à dos filhotes maiores, quando há escassez de recursos 
alimentares (BLOMQVIST et al., 1997; REED et al., 1999). Se tal estratégia é válida para avestruzes, ainda não existem evidências. A falta de diferenças no peso vivo e na morfometria aos 15 dias de idade, de filhotes oriundos de ovos de diferentes pesos, pode ser o resultado vantajoso da domesticação, quando é oferecido um aporte de recursos alimentares previsíveis e de alta qualidade, suprindo possíveis deficiências que se manifestariam em um ambiente natural.

Os achados deste estudo com avestruzes estão em contradição ao previsto e observado em aves precociais (BLOMQVIST et al., 1997; GÖTH \& EVANS, 2004), especialmente em outra ratita como o emu, em que o peso dos ovos se correlacionou com o maior peso e os maiores tibiotarsos dos filhotes (DZIALOWSKI \& SOTHERLAND, 2004). O tamanho do ovo relacionado ao maior tamanho de filhotes nas aves precociais está relacionado ao maior conteúdo da gema em relação aos demais constituintes do ovo (DZIALOWSKI \& SOTHERLAND, 2004). A relação gema/outros constituintes em emus é de 0,47 e em avestruzes é de 0,38 (DZIALOWSKI \& SOTHERLAND, 2004). Alternativamente, a água do albúmem pode ser crítica na relação peso do ovo/peso vivo do filhote (DZIALOWSKI \& SOTHERLAND, 2004), resultando em um maior volume sanguíneo do filhote ao nascer. Mesmo sob o aparente paradoxo para uma ave considerada precocial, a avestruz possui um dos menores ovos em relação ao tamanho do corpo, o que possivelmente poderia explicar a alta taxa de mortalidade infantil nesta espécie (DEEMING \& AR, 1999).

Alguns autores, como DEEMING \& AR (1999) e ZOCCARATO et al. (2004), relataram que os filhotes de avestruzes, ao eclodirem, possuem um peso que se correlaciona ao peso dos ovos. Os dados do presente estudo foram mensurados no 15 ia póseclosão, tempo que pareceu suficiente para que as características do ovo, refletidas no peso, não interferissem no peso vivo dos filhotes.

Nossos resultados indicam que os filhotes de avestruzes criados no sistema deste criadouro padrão do Distrito Federal são muito mais precoces do que o relatado por ZOCCARATO et al. (2004), quando a relação peso do ovo/peso do filhote somente desapareceu na oitava semana de vida. Os menores pesos dos filhotes observados, concomitantemente à falta de correlação entre peso do ovo/peso do filhote em nosso estudo, aos 15 dias, quando comparado a outros estudos, pode ser um indício de que há um problema de manejo da cria. O peso do filhote é representado em maior parte pelo balanço entre a energia lipídica herdada no ovo e a perda de energia que ocorre nas primeiras semanas de vida (SUPERCHI et al., 2002). Em avestruzes, a reserva representada pela gema residual pode permanecer no filhote até a terceira semana de idade, esgotando-se proporcionalmente à rapidez com que a ave ingere alimentos (VERWOERD et al., 1999). Um baixo aporte de lipídeos da reprodutora, ou um dispêndio excessivo de energia pelo filhote, podem explicar a falta de relação do peso do ovo e a discrepância do peso de filhotes criados no presente modelo de manejo. Considerando-se que os ovos tiveram pesos médios semelhantes aos pesos médios relatados em outros estudos (SUPERCHI et al.,2002; ZOCCARATO et al., 2004; ESTRADA et al., 2006), não é razoável interpretar-se que o problema está no aporte de nutrientes pela fêmea reprodutora.

Considerando as outras ratitas, DI CAMPOS et al. (2005) não encontraram uma relação expressiva entre o peso dos ovos e o peso dos filhotes de emas (Rhea americana), informando que esta relação estaria em torno de 65 a 70\%. Contudo, em emus (Dromaius novaehollandiae), o investimento parental no tamanho do ovo possui uma clara relação com o tamanho dos filhotes ao nascerem (DZIALOWSKI \& SOTHERLAND, 2004).

BLOMQVIST et al. (1997) realizaram uma revisão comparando o tamanho dos ovos, o cuidado parental e a sobrevivência de filhotes em 14 estudos em que houve a troca de ovos e a incubação adotiva. Esses autores demonstraram que somente em quatro estudos houve unicamente o efeito do tamanho dos ovos influenciando a sobrevivência dos filhotes. Em outros oito estudos, o cuidado parental, juntamente com o tamanho dos ovos, parece ser sinérgico para o sucesso da sobrevida dos filhotes. Então, as espécies em que apenas o tamanho dos ovos determina a qualidade dos filhotes parecem ser mais exceções do que uma norma.

\section{CONCLUSÃO}

Não houve uma relação entre o tamanho dos ovos incubados artificialmente e as dimensões dos filhotes de avestruzes aos 15 dias de idade. O possível custo energético da mãe, representado no tamanho dos ovos, desvanece-se até a segunda semana de vida de avestruzes criados artificialmente. Não houve diferenças significativas entre as medidas morfométricas e o tamanho do ovo. O crescimento das avestruzes investigadas neste criatório comercial está de acordo com o padrão da espécie e o peso dos animais estava intimamente correlacionado com as demais medidas morfométricas (tarso-metatarso direito e esquerdo e altura da cernelha). O tamanho do ovo aparentemente não possui importância para explicar 
possíveis diferenças quanto ao desenvolvimento dos filhotes de avestruzes aos 15 dias de idade.

\section{AGRADECIMENTOS}

Ao médico veterinário Belarmino Gama, pela gentil cessão dos animais e das instalações para o desenvolvimento do estudo; e ao Decanato de Pesquisa e Pós-graduação da UnB e à FINATEC/UnB, que financiaram parcialmente o projeto. A. B. B. Alvarenga foi apoiado com uma bolsa de Mestrado do CNPq.

\section{REFERÊNCIAS}

ANDERSON, V. R.; ALISAUSKAS, R. T. Egg size, body size, locomotion, and feeding performance in captive King Eiders ducklings. Condor, Albuquerque, v.103, p.195-199, 2001.

BLOMQVIST, D., et al. Parental quality and egg size affect chick survival in a precocial bird, the lapwing Vanellus vanellus. Oecologia, Berlin, v.110, p.18-24, 1997.

BOLTON, M. Determinants of chick survival in the lesser black backed gull; relative contributions of egg-size and parental quality. Journal of Animal Ecology, Oxford, v.60, p.949960, 1991.

CHRISTIANS, J. K. Avian egg size: variation within species and inflexibility within individuals. Biological Reviews, Cambridge, v.77, p.1-26, 2002.

DEEMING, D. C.; AR, A. Factors affecting success of commercial incubation. In: DEEMING, D. C. The Ostrich: biology, production and health, Cambridge: CABI, 1999. p.159-190.

DI CAMPOS, M. S., et al. Estimativa de correlações entre medidas morfométricas, peso do ovo e peso de filhotes de emas criados em cativeiro. Ciência Rural, Santa Maria, v.35, n.3, p.678-683, 2005.

DZIALOWSKI, E. M.; SOTHERLAND P. R. Maternal effects of egg size on emu Dromaius novaehollandiae egg composition and hatchling phenotype. Journal of Experimental Biology, Cambridge, v.207, p.597-606, 2004.

ESTRADA, M. A. J. et al. Effect of the initial weight and weight loss during incubation on the hatchability rate of ostrich eggs (Struthio camelus). Veterinaria México, Mexico, v.37, n.4, p.487-492, 2006.

FINKLER, M. S., et al. Experimental manipulation of egg quality in chickens: influence of albumen and yolk on the size and body composition of near-term embryos in a precocial bird. Journal of Comparative Physiology B, Berlin, v.168, p.17-24, 1998.

GONZALEZ, A., et al. Factors affecting ostrich egg hatchability. Poultry Science, Savoy, v.78, p.1257-1262, 1999.

GÖTH, A.; EVANS, C. S. Egg size in Australian brush-turkey Alectura Iathami hatchlings predicts motor performance and postnatal weight gain. Canadian Journal of Zoology, Ottawa, v.82, p.972-979, 2004.

HILL, W. L. Intraspecific variation in egg composition. Wilson Bulletin, Albuquerque, v.107, n.2, p.382-387, 1995.

KRIST, M., et al. Egg size and offspring performance in the collared flycatcher (Ficedula albicollis): a within-clutch approach. Oecologia, Berlin, v.140, p.52-60, 2004.

LINDSTRÖM, J. Early development and fitness in birds and mammals. Trends Ecology Evolution, London, v.14, p.343348, 1999.

MAGRATH, M. J. L., et al. J. Egg size and laying order in relation to offspring sex in the extreme sexually size dimorphic brown songlark, Cinclorhamphus cruralis. Behavioral Ecology Sociobiology, Berlin, n.54, p.240-248, 2003.

NAGER, R., et al. Within-clutch trade-offs between the number and quality of eggs: experimental manipulations in gulls. Ecology, Ithaca, v.81, p.1339-1350, 2000.

NOLAN J. R. V.; THOMPSON, C. F. Egg volume as a predictor of hatchling weight in the brown-headed crowbird. Wilson Bulletin, Albuquerque, v.90, n.3, p.353-358, 1978.

RAHN, H., et al. A. Relation of avian egg weight to body weight. Auk, Albuquerque, v.92, p.750-765, 1975.

REED, W. L., et al. Consequences of egg-size variation in the Red-winged Blackbird. Auk, Albuquerque, v.116, p.549-552, 1999.

RHYMER, J. M. The effect of egg size variability on thermoregulation of Mallard (Anas platyrhynchos) offspring and its implications for survival. Oecologia, Berlin, v.75, p.2024, 1988

SLATTERY, S.; ALISAUSKAS, R. T. Egg characteristics and body reserves of neonate Ross' and Lesser Snow geese. Condor, Albuquerque, v.97, p.970-985, 1995.

SUPERCHI, P., et al. Italian ostrich (Struthio camelus) eggs: physical characteristics and chemical composition. Annali della Facoltà di Medicina Veterinaria di Parma, Parma, v.22, p.155-162, 2002.

VERWOERD, D. J., et al. Rearing environments around the world. In: DEEMING, D. C. The ostrich: biology, production and health. Cambridge: CABI Publish, 1999. p.163-206.

WILLIAMS, T. D. Intraspecific variation in egg size and egg composition in birds: effects on offspring fitness. Biological Reviews, Cambridge, v.68, p.35-59, 1994.

ZOCCARATO, I. et al. Effect of egg weight on ostrich (Struthio camelus) chick weight and growth. Italian Journal of Animal Science, Bologna, v.3, p.7-17, 2004. 